\title{
Prática de atividade física e sedentarismo em brasileiros: resultados da Pesquisa Nacional por Amostra de Domicílios (PNAD) - 2008
}

\author{
Practice of physical activity and sedentarism among Brazilians: \\ results of the National Household Sample Survey - 2008
}

\author{
Alan Goularte Knuth ${ }^{1}$ \\ Deborah Carvalho Malta ${ }^{2}$ \\ Samuel Carvalho Dumith ${ }^{1}$ \\ Cimar Azeredo Pereira ${ }^{3}$ \\ Otaliba Libânio Morais Neto ${ }^{4}$ \\ Jose Gomes Temporão ${ }^{5}$ \\ Gerson Penna ${ }^{4}$ \\ Pedro Curi Hallal ${ }^{1}$
}

${ }^{1}$ Universidade Federal de Pelotas (UFPel). Programa de Pós-graduação em Epidemiologia. Rua Marechal Deodoro 1160/3 andar, Centro. 96020-220 Pelotas RS.

alan_knuth@yahoo.com.br ${ }^{2}$ Coordenação Geral de

Doenças e Agravos Não Transmissíveis, Secretaria de Vigilância em Saúde, Ministério da Saúde.

${ }^{3}$ Instituto Brasileiro de Geografia e Estatística.

${ }^{4}$ Secretaria de Vigilância em Saúde, Ministério da Saúde.

${ }^{5}$ Ministério da Saúde.
Abstract Population surveys are a key component of the relevant activities for public health. There is growing interest in identifying behavioral aspects which influence individual and collective health, such as physical activity (PA). The aim of this study is to present PA data from the 2008 Brazilian National Household Survey (PNAD) according to regional distribution and the socio-demographic characteristics. A partnership between the Brazilian Institute of Geography and Statistics and the Ministry of Health was responsible for preparing the health supplement of the questionnaire. The sample size was 292,553 individuals. The proportion of subjects practicing PA at the recommended levels in leisure time was $10.5 \%$, exactly the same proportion of subjects reporting active transportation to and from work. Males and younger adults were more active; schooling was directly related to leisure-time $P A$ and inversely associated with transport-related physical activity. One fifth of the Brazilians did not report any physical activity at all and $35.7 \%$ watch TV for 3 hours or more, on average, per day. These data may help design public policies aimed at promoting health, in particular through PA, which may positively influence on the quality of life of the Brazilian population.

Key words Physical activity, Survey, Public health
Resumo Inquéritos populacionais estão no centro das atividades relevantes para a saúde pública. Atualmente tem-se interesse em compreender aspectos comportamentais influentes na mudança do quadro de saúde individual e coletiva, entre eles a atividade física. O objetivo do presente estudo é apresentar os resultados de prática de atividade física (AF) da Pesquisa Nacional por Amostra de Domicílios (PNAD) em 2008, conforme distribuição regional e características sociodemográficas. O convênio firmado entre o IBGE e o Ministério da Saúde foi responsável pelo levantamento suplementar de saúde. O tamanho de amostra foi de 292.553 pessoas. A prevalência de AF nos níveis recomendados no lazer foi de 10,5\%, o mesmo percentual de indivíduos relatando deslocamento ativo para o trabalho. Homens e indivíduos mais jovens foram mais ativos e houve relação direta entre escolaridade e AF no lazer e inversa entre escolaridade e atividade física no deslocamento. Um em cada cinco brasileiros não pratica qualquer AF, e um em cada três assistem, em média, 3 h ou mais de televisão por dia. Estes dados visam apoiar as politicas públicas no desenho de estratégias que promovam ações sustentadas de promoção da saúde, especialmente de AF, visando $o$ alcance de resultados que influenciem positivamente na qualidade de vida da população.

Palavras-chave Atividade física, Inquérito, Saúde pública 


\section{Introdução}

Inquéritos populacionais estão no centro das atividades relevantes e de produção consistente de conhecimento para a saúde pública. Tais pesquisas permitem que sejam construídos indicadores de saúde e de doença e comportamentos de risco ou de proteção, e que sejam avaliados os determinantes sociais de saúde/doença ${ }^{1}$. Com base em tais dados, o conjunto de esferas responsáveis pela produção de conhecimento e criação de políticas públicas no país pode compreender os aspectos de saúde/doença em termos de magnitude e distribuição. A organização sistemática de inquéritos reflete as mudanças temporais da situação de saúde e permite o monitoramento de determinado aspecto relevante para o contexto avaliado. Quando pensados sob o ponto de vista de mudança na situação de saúde, os inquéritos são parâmetros importantes para a avaliação direta ou indireta de políticas públicas e para situá-las em termos de mudanças nas condições de saúde e de qualidade de vida das populações.

Em uma perspectiva atual, no âmbito da avaliação das condições de saúde, temos um expressivo interesse em compreender aspectos comportamentais, influentes na mudança do quadro de saúde individual e coletivo. Nessa lógica tem-se tornado consistente o estudo da prática de atividade física, dada sua importante relação com eventos de saúde e qualidade de vida em jovens, adultos e idosos ${ }^{2}$.

Pelo recente foco de abordagem em atividade física nos inquéritos de saúde, as informações referentes a este tema são também extremamente recentes. Em 1996-1997, a Pesquisa de Padrões de Vida (PPV) avaliou a atividade física de lazer por meio de um breve questionário. Menos de $5 \%$ dos entrevistados relataram praticar atividade física de lazer nos níveis recomendados ${ }^{3}$. A avaliação de atividade física, por meio de questionário específico e detalhado, com base de dados nacional ( 15 capitais + Distrito federal), teve abordagem pioneira em 2002-2003 no Inquérito Domiciliar sobre Comportamentos de Risco e Morbidade Referida de Doenças e Agravos Não Transmissíveis, sob coordenação do Instituto Nacional do Câncer (INCA) e Ministério da Saúde . Também nesse período, a investigação epidemiológica do tema atividade física/inatividade físi$\mathrm{ca} /$ sedentarismo passou a ser crescente e realizado com abordagem bastante variada em termos de instrumentos, faixa-etária e domínio da atividade física avaliado ${ }^{5,6}$. Tanto inquéritos populacionais quanto estudos locais pontuais apontam para uma baixa prática de atividade física, no lazer, por adultos e adolescentes ${ }^{3,4}$.

Com a epidemiologia da atividade física passando a ocupar um espaço de destaque nas avaliações em saúde, o Ministério da Saúde, o IBGE, as universidades e os centros colaboradores consolidaram outros mecanismos de conhecimento dos padrões de atividade física de brasileiros e passaram também monitorar as mudanças temporais nesse comportamento. Assim, a Vigilância de Fatores de Risco e Proteção para Doenças Crônicas por Inquérito Telefônico (Vigitel) ${ }^{7}$ e a Pesquisa Nacional de Saúde do Escolar $(\mathrm{PeNSE})^{8}$ foram desenhadas para mapear situações de saúde em diversos aspectos, entre eles a atividade física de adultos e jovens escolares, respectivamente.

Em moldes semelhantes, a Pesquisa Nacional por Amostra de Domicílios (PNAD), criada em 1967 para avaliar o desenvolvimento socioeconômico do país, incluiu em determinados levantamentos, suplementos específicos de saúde como nos anos de 1998, 2003 e 2008. No levantamento suplementar de saúde em 2008 a prática de atividade física foi avaliada pela primeira vez. O que traz uma informação adicional aos inquéritos anteriores e aumenta a cobertura de medição de atividade física em relação aos demais inquéritos populacionais, pois a abrangência da PNAD é ainda maior.

O objetivo do presente estudo é apresentar os resultados de prática de atividade física da PNAD em 2008, conforme distribuição regional e características sociodemográficas. Também são discutidos no presente manuscrito os resultados para diferentes domínios de atividade física e o hábito de assistir televisão na população brasileira.

\section{Metodologia}

Em 2008, o convênio firmado entre o IBGE e o Ministério da Saúde foi responsável pelo levantamento suplementar de saúde, abordado pela terceira vez na PNAD, com intervalos regulares de cinco anos. A PNAD é realizada por meio de amostra probabilística de domicílios, a partir de estágios múltiplos de seleção, a saber: unidades primárias ou municípios, unidades secundárias ou setores censitários e unidades terciárias ou domicílios. Os municípios foram classificados em duas categorias, autorrepresentativos e não autorrepresentativos, os últimos passaram por um processo de estratificação e, em cada estrato, foram selecionados com reposição e probabilidade proporcional à população obtida no Censo 
Demográfico de 2000. Nos setores censitários, se utilizou a probabilidade proporcional e também com reposição. Os domicílios foram selecionados com equiprobabilidade. Maiores detalhes sobre a amostragem e outros aspectos da PNAD $^{9}$ podem ser encontrados em seu sítio na Internet.

Cerca de 2500 entrevistadores foram os responsáveis pela coleta de dados da PNAD 2008, por meio de coletor eletrônico. O suplemento saúde da PNAD no ano de 2008, com 163 questões, incluiu diversos aspectos, entre os quais a prática de atividade física, a qual foi estudada em moradores com 14 anos ou mais de idade. Por meio de oito questões procurou-se avaliar a atividade física nos seguintes domínios: deslocamento para o trabalho, atividade laboral, realização de faxina no ambiente doméstico e atividades físicas de lazer. No mesmo bloco, uma questão avaliou a frequência de horas sentado assistindo televisão, em todos moradores da casa.

As questões sobre atividade física e o hábito de assistir televisão foram construídas tendo por base os questionários do Vigite ${ }^{10}$. No entanto, existem algumas diferenças, especialmente em função da coleta de dados por entrevista face a face (PNAD) em comparação às entrevistas telefônicas (Vigitel). O questionário da $\mathrm{PNAD}^{11}$ também está disponível para acesso público no sítio eletrônico do IBGE. No instrumento sobre atividade física, foi questionado se os indivíduos costumam ir a pé ou de bicicleta para o trabalho e o tempo gasto; também foi perguntado se o indivíduo, em seu trabalho, anda a maior parte do tempo, carrega peso ou faz esforço intenso; sobre a atividade física no domicílio foi indagado se o indivíduo faz faxina, e se sim, se essa limpeza é individual ou com ajuda de outra pessoa; ainda quatro questões abordam aspectos referentes a exercício fisco ou esportes, como as modalidades escolhidas, frequência de dias e tempo gasto nas práticas.

As variáveis dependentes utilizadas nesse artigo são:

- ativo no deslocamento: indivíduo empregado que costuma ir a pé ou de bicicleta de casa para o trabalho, com trajeto de 30 minutos ou mais somando a ida e a volta;

- ativo no lazer: indivíduo que pratica atividades físicas leves ou moderadas em 5 dias ou mais na semana por pelo menos 30 minutos e/ ou indivíduos que praticam atividades físicas vigorosas em 3 dias ou mais na semana por pelo menos 20 minutos;

- inativos: indivíduo que relatou não praticar qualquer atividade física em todos os domínios estudados (deslocamento para o trabalho, atividade laboral, faxina no ambiente doméstico e atividades físicas de lazer);

- hábito de assistir TV: indivíduo que assiste televisão sentado por 3 horas ou mais diariamente, fora do trabalho.

As variáveis independentes utilizadas neste artigo foram: grandes regiões geográficas (Nordeste, Norte, Sudeste, Sul e Centro-Oeste), sexo (masculino ou feminino), grupos de idade (anos completos) e escolaridade (conforme anos completos de estudo). O indicador ativo no lazer também foi descrito conforme unidades federativas brasileiras.

As análises foram conduzidas no Programa Stata, versão 9.0. A amostra de 391.868 pessoas e 150.591 domicílios foi expandida para a população brasileira e o efeito de delineamento amostral foi considerado nas análises, por meio do grupo de comandos svy. Atribuiu-se também um peso para cada indivíduo, utilizando-se o comando "fweight". A expansão amostral é baseada na projeção da população residente de cada Unidade da Federação, considerando a evolução populacional entre os Censos de 1991 e 2000 e a contagem da população em 2007. Apesar de todas as associações terem sido testadas por meio do teste do qui-quadrado, os valores $\mathrm{p}$ (todos altamente significativos) não foram mencionados no corpo do texto ou nas tabelas e figuras, em função do elevado poder amostral.

\section{Resultados}

A amostra analisada neste estudo ficou constituída por 292.553 indivíduos com 14 anos ou mais. Usando o fator de ponderação para frequência da população brasileira, o $\mathrm{N}$ total corresponde a 142.533.480 pessoas. Entre os entrevistados, quase metade $(43,5 \%)$ residiam na região Sudeste, $51,5 \%$ eram do sexo feminino, 8,8\% tinham 65 anos ou mais de idade e $54,6 \%$ possuíam o ensino fundamental completo (Tabela 1).

A prevalência de indivíduos classificados com ativos no lazer foi exatamente a mesma daqueles considerados ativos no deslocamento, correspondendo a 10,5\% da população. Aproximadamente um quinto da população $(20,2 \%)$ relatou não praticar nenhuma atividade física, seja no trabalho, lazer, deslocamento ou domicílio. Com relação ao hábito de assistir televisão, 35,7\% referiu fazê-lo em média por pelo menos 3 horas por dia.

A Tabela 2 apresenta a prática de atividade física no deslocamento para o trabalho e no lazer 
conforme região do país e variáveis sociodemográficas. A prevalência de deslocamento ativo foi maior para as regiões Nordeste $(15,4 \%)$ e Norte

Tabela 1. Descrição de variáveis independentes conforme grandes regiões e informações sociodemográficas em indivíduos de 14 anos ou mais. PNAD - 2008.

\begin{tabular}{lrr}
\hline Característica & \multicolumn{1}{c}{$\mathbf{N}$} & \multicolumn{1}{c}{$\%($ IC95\%) } \\
& expandido & \\
\hline Região do país & & \\
$\quad$ Norte & 10.612 .146 & $7,5(6,9-8,0)$ \\
$\quad$ Nordeste & 38.519 .829 & $27,0(26,6-27,4)$ \\
Sudeste & 61.965 .524 & $43,5(43,0-44,0)$ \\
Sul & 21.157 .176 & $14,8(14,5-15,2)$ \\
Centro-Oeste & 10.278 .805 & $7,2(7,0-7,4)$ \\
Sexo & & \\
Masculino & 68.727 .943 & $48,2(48,1-48,4)$ \\
Feminino & 73.805 .537 & $51,8(51,6-51,9)$ \\
Idade (anos) & & \\
14 a 24 & 36.804 .742 & $25,8(25,6-26,0)$ \\
25 a 34 & 30.549 .811 & $21,4(21,2-21,6)$ \\
35 a 44 & 26.595 .887 & $18,7(18,5-18,8)$ \\
45 a 54 & 21.857 .886 & $15,3(15,2-15,5)$ \\
55 a 64 & 14.193 .563 & $10,0(9,8-10,1)$ \\
65 ou mais & 12.531 .591 & $8,8(8,6-9,0)$ \\
Escolaridade (anos) & & \\
0 a 8 & 64.719 .420 & $45,4(44,9-45,9)$ \\
9 a 11 & 26.976 .193 & $18,9(18,7-19,2)$ \\
12 ou mais & 50.837 .867 & $35,7(35,2-36,1)$ \\
Total & $\mathbf{1 4 2 . 5 3 3 . 4 8 0}$ & $\mathbf{1 0 0 , 0}$ \\
\hline & &
\end{tabular}

$(11,9 \%)$, sexo masculino $(11,4 \%)$ e indivíduos entre 14 e 24 anos (11,5\%). Já a prevalência de ativos no lazer foi maior para a região CentroOeste $(12,0 \%)$, sexo masculino (12,7\%) e indivíduos entre 14 e 24 anos (16,1\%). A prevalência de indivíduos ativos no lazer conforme unidade federativa está ilustrada na Figura 1. A proporção de ativos oscilou de 5,8\% em Alagoas a 16,6\% no Distrito Federal.

A Figura 2 mostra a prática de atividade física no lazer e deslocamento de acordo com a escolaridade. Enquanto a escolaridade e a atividade física de lazer estão diretamente relacionadas, a associação é inversa entre escolaridade e atividade física no deslocamento.

Os resultados de inatividade física e hábito de assistir televisão 3 horas ou mais por dia conforme região do país e variáveis sociodemográficas estão apresentados na Tabela 3. O Sudeste foi a região com maior prevalência de inativos $(22,1 \%)$, enquanto a região Sul obteve o menor percentual $(17,4 \%)$. Os homens foram mais inativos que as mulheres ( $25,6 \%$ versus $15,2 \%)$, e a prevalência de inatividade física entre os idosos (65 anos ou mais) foi superior ao dobro daquela verificada nas demais faixas etárias. Indivíduos com maior escolaridade (12 anos ou mais) foram mais inativos do que os seus pares. Já o hábito de assistir TV foi mais frequente na região Sudeste $(38,3 \%)$, sexo feminino $(38,7 \%)$, indivíduos mais jovens $(43,4 \%)$ e naqueles com 9 a 11 anos de estudo $(41,1 \%)$.

Tabela 2. Prática de atividade física no deslocamento para o trabalho e no lazer conforme região do país e variáveis sociodemográficas em indivíduos de 14 anos ou mais. PNAD - 2008.

\begin{tabular}{|c|c|c|c|c|}
\hline & \multicolumn{2}{|c|}{ Ativo no deslocamento } & \multicolumn{2}{|c|}{ Ativo no lazer } \\
\hline & $\mathrm{N}$ expandido & $\%(\mathrm{IC} 95 \%)$ & $\mathrm{N}$ expandido & $\%$ \\
\hline \multicolumn{5}{|l|}{ Região do país } \\
\hline Norte & 759.082 & $11,9(10,4-13,6)$ & 1.145 .405 & $10,8(10,1-11,5)$ \\
\hline Nordeste & 3.484 .084 & $15,4(14,5-16,4)$ & 3.825 .287 & $9,9(9,5-10,3)$ \\
\hline Sudeste & 3.240 .413 & $8,6(8,2-9,1)$ & 6.330 .017 & $10,2(9,9-10,5)$ \\
\hline Sul & 1.227 .574 & $9,0(8,3-9,7)$ & 2.398 .627 & $11,3(10,8-12,0)$ \\
\hline Centro-Oeste & 433.008 & $6,8(6,2-7,4)$ & 1.230 .928 & $12,0(11,4-12,5)$ \\
\hline \multicolumn{5}{|l|}{ Sexo } \\
\hline Masculino & 5.758 .794 & $11,4(10,9-11,2)$ & 8.693 .642 & $12,7(12,4-12,9)$ \\
\hline Feminino & 3.395 .367 & $9,4(9,0-9,8)$ & 6.236 .622 & $8,5(8,2-8,7)$ \\
\hline \multicolumn{5}{|l|}{ Idade (anos) } \\
\hline 14 a 24 & 2.041 .210 & $11,5(10,9-12,1)$ & 5.928 .089 & $16,1(15,7-16,5)$ \\
\hline 25 a 34 & 2.326 .371 & $10,1(9,6-10,5)$ & 3.028 .219 & $9,9(9,6-10,2)$ \\
\hline 35 a 44 & 2.110 .437 & $10,3(9,9-10,8)$ & 2.094 .636 & $7,9(7,6-8,2)$ \\
\hline 45 a 54 & 1.644 .567 & $10,7(10,2-11,2)$ & 1.669 .128 & $7,6(7,3-8,0)$ \\
\hline 55 a 64 & 764.192 & $10,5(9,9-11,1)$ & 1.259 .427 & $8,9(8,5-9,3)$ \\
\hline 65 ou mais & 267.384 & $10,1(9,2-11,1)$ & 950.765 & $7,6(7,2-8,0)$ \\
\hline Total & 9.154 .161 & $10,5(10,2-10,9)$ & 14.930 .264 & $10,5(10,3-10,7)$ \\
\hline
\end{tabular}


Figura 1. Prevalência de ativos no lazer em indivíduos com 14 anos ou mais para cada estado do país. PNAD - 2008.

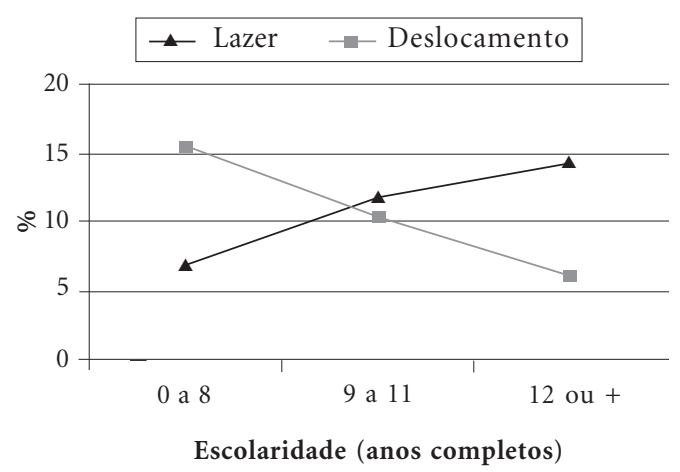

Figura 2. Prevalência de atividade física nos níveis recomendados no lazer e deslocamento ativo conforme a escolaridade, em indíviduos com 14 anos ou mais. PNAD - 2008 .

\section{Discussão}

Esse é o primeiro inquérito populacional de saúde a descrever a prevalência de atividade física em amostra representativa de todo o Brasil e com faixa etária ampliada, captando adolescentes, adultos e idosos. Apenas 10,5\% dos brasileiros com 14 anos ou mais de idade cumprem as reco- mendações para atividade física de lazer². Esse comportamento foi mais frequente em indivíduos de maior escolaridade, exatamente o oposto ao observado para atividade física no deslocamento para o trabalho, a qual é marcada como um comportamento de jovens e indivíduos de baixa escolaridade. A inatividade física foi de $20,2 \%$ e o hábito de assistir pelo menos 3 horas de televisão foi um comportamento bastante comum na população brasileira.

A investigação separada de atividade física por domínios (deslocamentos, atividades domésticas, trabalho e no período de lazer) é bastante referendada na literatura científica ${ }^{12,13}$. Essa distinção, além de didática, é importante para a captação de informações com maior qualidade nos instrumentos pertinentes à área. Além disso, essa separação contribui bastante para esclarecer a presença de um marcador social envolvendo a atividade física no deslocamento e no lazer. A atividade física realizada no deslocamento para o trabalho é mais comum nas regiões mais empobrecidas do país (Norte e Nordeste), em homens, pessoas mais jovens e com baixa escolaridade. Ao que tudo indica esse tipo de atividade física não é realizada como opção de promoção da qualidade de vida, e sim, fundamentada basicamente em uma necessidade. Ainda que o deslocamento ativo possa tra- 
Tabela 3. Inatividade física e hábito de assistir televisão 3 horas ou mais por dia, em média, conforme região do país e variáveis sociodemográficas em indivíduos de 14 anos ou mais. PNAD - 2008.

\begin{tabular}{|c|c|c|c|c|}
\hline & \multicolumn{2}{|c|}{ Inativo } & \multicolumn{2}{|c|}{ Assiste TV $3 \mathrm{~h} / \mathrm{mais}$ por dia } \\
\hline & $\mathrm{N}$ expandido & $\%$ & $\mathrm{~N}$ expandido & $\%$ \\
\hline \multicolumn{5}{|l|}{ Região do país } \\
\hline Norte & 1.961 .014 & $18,5(17,5-19,5)$ & 3.550 .841 & $33,5(31,7-35,3)$ \\
\hline Nordeste & 7.392 .937 & $19,2(18,6-19,8)$ & 13.534 .598 & $35,1(33,9-36,4)$ \\
\hline Sudeste & 13.696 .363 & $22,1(21,7-22,5)$ & 23.705 .605 & $38,3(37,5-39,0)$ \\
\hline Sul & 3.675 .582 & $17,4(16,7-18,1)$ & 6.582 .826 & $31,1(30,1-32,2)$ \\
\hline Centro-Oeste & 2.064 .434 & $20,1(19,3-20,9)$ & 3.565 .773 & $34,7(33,5-35,9)$ \\
\hline \multicolumn{5}{|l|}{ Sexo } \\
\hline Masculino & 17.571 .668 & $25,6(25,1-26,0)$ & 22.369 .364 & $32,6(32,0-33,1)$ \\
\hline Feminino & 11.212 .662 & $15,2(14,9-15,5)$ & 28.570 .279 & $38,7(38,2-39,3)$ \\
\hline \multicolumn{5}{|l|}{ Idade (anos) } \\
\hline 14 a 24 & 7.216 .662 & $19,6(19,2-20,1)$ & 15.977 .665 & $43,4(42,7-44,1)$ \\
\hline 25 a 34 & 5.112 .385 & $16,7(16,3-17,1)$ & 10.393 .025 & $34,0(33,4-34,7)$ \\
\hline 35 a 44 & 4.497 .596 & $16,9(16,5-17,3)$ & 8.032 .872 & $30,2(29,6-30,9)$ \\
\hline 45 a 54 & 4.051 .323 & $18,5(18,1-19,0)$ & 6.812 .875 & $31,2(30,5-31,9)$ \\
\hline 55 a 64 & 3.128 .950 & $22,0(21,4-22,6)$ & 4.826 .122 & $34,0(33,2-34,8)$ \\
\hline 65 ou mais & 4.783 .414 & $38,2(37,4-39,0)$ & 4.897 .084 & $39,1(38,2-40,0)$ \\
\hline \multicolumn{5}{|c|}{ Escolaridade (anos) } \\
\hline 0 a 8 & 12.511 .700 & $19,3(19,0-19,7)$ & 22.411 .914 & $34,6(33,9-35,3)$ \\
\hline 9 a 11 & 4.832 .113 & $17,9(17,5-18,3)$ & 11.093 .197 & $41,1(40,5-41,8)$ \\
\hline 12 ou mais & 11.446 .517 & $22,5(22,1-22,9)$ & 17.434 .532 & $34,3(33,8-34,9)$ \\
\hline Total & 28.790 .330 & $20,2(19,9-20,5)$ & 50.939 .643 & $35,7(35,2-36,3)$ \\
\hline
\end{tabular}

zer benefícios para a saúde, ${ }^{14}$ ir a pé ou caminhando para o trabalho na realidade brasileira parece indicar falta de outra opção e não uma escolha individual para promoção da saúde. A determinação da atividade física envolve diversos componentes complexos. A utilização de aspectos individuais, como sexo, idade e escolaridade, não afasta a necessidade de se atentar para questões mais amplas e que dependem fortemente de políticas públicas, responsabilizando diversas esferas. A escolha individual por comportamentos ativos está intimamente atrelada às condições de vida, saúde, lazer, e, por isso, a atividade física é resultante de uma configuração ampla de vida, e em nosso estudo, investigamos apenas alguns aspectos isoladamente.

Não há recomendações consolidadas para a prática de atividade física no deslocamento isoladamente. A maioria dos órgãos de saúde prioriza a recomendação focando na atividade física total, sugerindo que o indivíduo realize 30 minutos de atividade física em cinco dias ou mais na semana. No período de deslocamento, consideramos ativos os indivíduos que relataram, costumeiramente, se deslocarem a pé ou de bicicleta para o trabalho com trajeto total igual ou superior a $30 \mathrm{minu}$ tos diários. Somando os dois domínios (lazer e deslocamento), 19,1\% dos indivíduos foram ativos no lazer ou no deslocamento; um somatório simples das duas prevalências geraria $21 \%$, o que confirma que a prática de atividade física em um domínio independe do outro. Também por isso, as características associadas a um ou outro domínio de atividade física são diferentes.

Apenas 10,5\% dos brasileiros com 14 anos ou mais que cumprem as recomendações de atividade física no lazer ${ }^{2}$. O padrão aqui encontrado repete estudos populacionais anteriores ${ }^{15,16}$, onde homens, jovens e indivíduos de maior escolaridade são mais ativos, o que mais uma vez remete a um aspecto social, onde a atividade física no lazer, por muitas vezes, encontra-se ligada a entidades privadas e assim restrita a certos grupos populacionais. Estratégias públicas de promoção da atividade física na comunidade têm aumentado recentemente em nosso país ${ }^{17,18} \mathrm{e}$ devem ser fortalecidas e encorajadas. A divulgação de experiências exitosas e articuladas com o sistema de saúde devem ser incentivadas e podem aproximar brasileiros de atividades físicas prazerosas e com significado próprio, de comunidades e contextos locais.

Em 2009, um total de $14,7 \%$ de indivíduos com 18 anos ou mais de idade foi considerado 
ativo no lazer, segundo o Vigitel, inquérito telefônico em capitais brasileiras e distrito federal. No presente estudo, a prevalência de ativos no lazer foi de 10,5\%, mesmo com faixa-etária mais abrangente, a partir dos 14 anos. Em nossa amostra, se restringirmos para a população de 18 anos ou mais, a prevalência seria de 9,4\% (no grupo de 14 a 17 anos a prevalência de ativos no lazer foi de 20,5\%). Estudos demonstram que a atividade física sofre redução exatamente no fim da adolescência $^{19}$ e esse é um período da vida em que o aconselhamento para a prática de atividade física deve ser fortalecido. Ainda que as prevalências de ativos no lazer não sejam idênticas entre Vigitel e PNAD, os sentidos dos resultados são muito semelhantes. No presente estudo a atividade física de lazer reduz gradualmente conforme aumenta o grupo de idade e sofre leve aumento na faixa de 55 a 64 anos, o mesmo ocorrendo no inquérito telefônico, assim como a maior atividade no sexo masculino e em indivíduos mais escolarizados. Deve-se considerar também que o Vigitel é restrito as capitais e a PNAD incluiu também cidades do interior, as quais podem ter padrões de comportamento que diferem dos grandes centros urbanos do país.

Para a inatividade física, os dados do Vigitel 2008 e PNAD foram 15,6\% e 20,2\%, respectivamente. As prevalências são próximas, entretanto algumas características de diferenciação entre os inquéritos, que podem auxiliar na identificação de particularidades entre estes, são mencionados: faixa-etária já discutida anteriormente, aperfeiçoamento no questionário do Vigitel em 2009 para inatividade física realizada no deslocamento, com a inserção de perguntas sobre atividades como ir ou vir à escola para jovens ou mães que levam ou buscam seus filhos na escola, não utilizadas na PNAD. Além disso, para se chegar ao indicador inatividade física, o procedimento é mais complexo, pois implica em avaliar quatro domínios (deslocamento para o trabalho, atividade laboral, faxina no ambiente doméstico e atividades físicas no lazer). Ou seja, são inúmeras questões, e qualquer alteração nas perguntas e na ordem das mesmas pode trazer prejuízo na comparabilidade entre diferentes estudos. Outro ponto a ser considerado consiste no fato da PNAD obter informação por meio do proxy, neste caso, sendo outro o informante, questões complexas como a variável inatividade física, podem acumular respostas imprecisas, que ajudam na explicação da diferença do resultado encontrado neste estudo comparado com o Vigitel, que pergunta diretamente ao informante ${ }^{7}$. Agrega-se esse ponto fundamental à diferença já mencionada na composição das amostras. Mesmo assim, as frequências podem ser consideradas bastante semelhantes entre os inquéritos.

Há uma elevada exposição a assistir televisão sentado, fora do ambiente de trabalho, por pelo menos 3 horas entre brasileiros. Para alguns autores o tempo excessivo em comportamento sedentário pode trazer consequências negativas para a saúde, como aumentar o risco de doenças cardiovasculares, independentemente da atividade física ${ }^{20}$. Esse campo de pesquisa, com abordagem nova, sugere que haja inclusive recomendações populacionais para que os indivíduos preocupem-se em não permanecer por longos períodos sentados. No presente estudo, os indivíduos com hábito de assistir televisão por mais tempo foram também mais inativos, em comparação àqueles que assistem menos tempo de televisão $(34,1 \%$ contra $30,7 \%$ de inatividade física respectivamente). Políticas de promoção da atividade física podem incluir mensagens de diminuição do comportamento sedentário, buscando alternativas de entretenimento, menos vinculadas ao comportamento sedentário e ao tempo assistindo televisão.

Dentre os limites do estudo destacamos a metodologia empregada na PNAD que obtém informação por meio de proxy, se por um lado isto reduz as perdas da pesquisa, aumenta a possibilidade de o informante familiar não repassar as informações de forma precisa de todos os familiares, podendo obter estimativas por vezes incorretas. Diversos produtos oriundos da PNAD analisaram os desfechos diretamente, sem avaliação rigorosa para quem foi o indivíduo respondente. Os próprios dados do relatório oficial não contemplam comparações entre respostas pessoais e por proxy.

Estes dados têm apoiado o Ministério da Saúde no sentido de desenvolver políticas públicas de promoção à saúde, fortalecendo essa estratégia no âmbito do Sistema Único de Saúde. Em 2006 foi publicada a Política Nacional de Promoção da Saúde ${ }^{18}$ e dentre suas prioridades foi inserido o tema da atividade física, o que tem resultado em ações e agendas concretas. Foi estruturado um sistema de monitoramento de fatores de risco de proteção a doenças crônicas não transmissíveis, o que incluí o monitoramento dos indicadores referentes à prática da atividade física. Neste sentido, a realização da PNAD e do VIGI$\mathrm{TEL}^{7}$ compõe este sistema de acompanhamento e tem possibilitado o estabelecimento de metas acordadas com os gestores no Pacto pela Vida ${ }^{18}$. 
Além disto, o Ministério da Saúde financiou nos últimos cinco anos cerca de mil municípios que vem desenvolvendo projetos locais de promoção à atividade física, inserindo a promoção da saúde como tema prioritário no SUS. Ainda tornase um grande desafio abordar o tema da promoção da saúde na sua complexidade, o que remete às articulações intersetoriais, visando a reorga-

\section{Colaboradores}

AG Knuth liderou a escrita e revisão do artigo. DC Malta definiu o plano de análise e a comunicação entre IBGE e Ministério da Saúde na identificação e circulação do banco de dados, juntamente com CA Pereira, o qual acompanhou a coleta. SC Dumith coordenou as análises do artigo. OL Morais Neto, JG Temporão e G Penna apoiaram a execução do trabalho de forma institucional. PC Hallal revisou a escrita do artigo em todas as etapas. nização dos espaços urbanos, favorecendo a mobilidade urbana e a prática do lazer ativo. Estes dados visam apoiar as políticas públicas no desenho de estratégias que promovam ações sustentadas de promoção da saúde, especialmente de atividade física, visando o alcance de resultados que impactem positivamente na qualidade de vida da população.

\section{Referências}

1. Viacava F. Informações em saúde: a importância dos inquéritos populacionais. Cien Saude Colet 2002;7(4):607-621.

2. Organização Mundial da Saúde. Global recommendations on Physical Activity for Health. Geneva: OMS; 2010.

3. Monteiro C, Conde W, Matsudo S, Matsudo VR, Bonseñor IM, Lotufo PA. A descriptive epidemiology of leisure-time physical activity in Brazil, 19961997. Rev Panam Salud Publica 2003;14(4):246-254.

4. Brasil. Ministério da Saúde (MS). Inquérito domiciliar sobre comportamentos de risco e morbidade referida de doenças e agravos não transmissíveis. Rio de Janeiro: INCA; 2003.

5. Hallal PC, Dumith SC, Bastos JP, Reichert FF, Siqueira FV, Azevedo MR. Evolution of the epidemiological research on physical activity in Brazil: a systematic review. Rev. Saude Publica 2007; 41(3):453-460.

6. Dumith SC. Physical activity in Brazil: a systematic review. Cad. Saude Publica 2009; 25(Suppl. 3):S41526.

7. Brasil. Vigitel Brasil 2008: Vigilância de fatores de risco e proteção para doenças crônicas por inquérito telefônico. Brasília: Ministério da Saúde, Secretaria de Vigilância em Saúde, Secretaria de Gestão Estratégica e Participativa; 2009.

8. Instituto Brasileiro de Geografia e Estatística (IBGE). Pesquisa Nacional de Saúde do Escolar. Rio de Janeiro: IBGE; 2009.

9. Instituto Brasileiro de Geografia e Estatística (IBGE) Pesquisa Nacional por Amostra de Domicílios. Um panorama da Saúde no Brasil. Acesso e Utilização de Serviços, Condições de Saúde e Fatores de Risco e proteção à Saúde 2008. Ministério do Planejamento 2010. [acessado 2010 out 13]. Disponível em: http://www.ibge.gov.br/home/estatistica/populacao/ panorama_saude_brasil_2003_2008/default.shtm.

10. Brasil. Ministério da Saúde (MS). Secretaria de Vigilância em Saúde. [acessado 2010 out 13]. Disponível em: http://portal.saude.gov.br/portal/arquivos/ pdf/167_ Q2008.pdf

11. Brasil. Instituto Brasileiro de Geografia e Estatística (IBGE). Pesquisa Nacional por Amostra de Domicílios. Rio de Janeiro: IBGE; 2008. [2010 out 13]. Disponível em: http://www.ibge.gov.br/home/estatistica/ populacao/panorama_saude_brasil_2003_2008/qstp nadsaude2008.pdf 
12. Florindo AA, Hallal PC, Moura EC, Malta DC. Practice of physical activities and associated factors in adults, Brazil, 2006. Rev Saude Publica 2009; 43(Supl. 2):65-73.

13. Malta DC, Moura EC, Castro AM, Cruz DKA, Morais Neto OL, Monteiro CA. Padrão de atividade física em adultos brasileiros: resultados de um inquérito por entrevistas telefônicas, 2006. Epidemiologia e Serviços de Saúde 2009; 18(1):7-16.

14. Hamer M, Chida Y. Active commuting and cardiovascular risk: a meta-analytic review. Preventive medicine 2008; 46(1):9-13.

15. Martins TG, Assis MA, Nahas MV, Gauche H, Moura EC. Leisure-time physical inactivity in adults and factors associated. Rev Saude Publica 2009; 43(5):814824.

16. Dias-da-Costa JS, Hallal PC, Wells JC, Daltoé T, Fuchs SC, Menezes AM, Olinto MT. Epidemiology of leisure-time physical activity: a population-based study in southern Brazil. Cad Saude Publica 2005; 21(1):275-282.

17. Knuth A, Malta D, Cruz D, Castro AM, Fagundes J, Sardinha LM, Gosch CS, Simões EJ, Hallal PC. Description of the countrywide physical activity network coordinated by the Brazilian Ministry of Health: 2005-2008. J Phys Act Health 2010; 7(2):253248.

18. Malta DC, Castro AM, Cruz DKA, Gosh CS. A Promoção da Saúde e da Atividade Física no Sistema Único de Saúde. Rev. bras. ativ. fis. saúde 2008; 13(1):24-27.

19. Gordon-Larsen P, Nelson MC, Popkin BM. Longitudinal physical activity and sedentary behavior trends: adolescence to adulthood. American journal of preventive medicine 2004; 27(4):277-283.

20. Hamilton MT, Healy GN, Dunstan DW, Zderic TW, Owen N. Too Little Exercise and Too Much Sitting: Inactivity Physiology and the Need for New Recommendations on Sedentary Behavior. Current Cardiovascular Risk Reports 2008; 2(4):292-298. 